\title{
Outcome of Microscopic-assisted Thyroid Surgery: An Institutional Retrospective Study
}

\author{
${ }^{1}$ Harendra Kumar Gautam, ${ }^{2}$ Devendra Maurya, ${ }^{3}$ Sanjay Sharma
}

\section{ABSTRACT}

Background: Microscopic-assisted thyroid surgery approach helps surgical performance and prevents complications.

Aims and Objectives: The aim of the present retrospective study was to review the thyroid surgery performed by using microscope in a single institution and analyze the outcomes with respect to those reported in the literature.

Materials and Methods: A total of 30 patients were included with more than 11 years of age presented with thyroid nodule, and thyroidectomy was done under microscopic vision.

Results: Majority of the patients were between 21 years and 40 years of age. Female:male ratio was about 5.6:1. Swelling in front of the neck was the most common presentation. Most common solitary thyroid swelling was the colloid goiter. Commonest surgery performed was hemithyroidectomy $(93.34 \%)$. Among the 30 patients, only two patients $(0.6 \%)$ had unilateral vocal fold immobility treated with medical therapy, phoniatric and neck physiotherapy. All two patients showed complete laryngeal recovery of motility within $6-8$ weeks after treatment. There were no cases of permanent unilateral or bilateral vocal cord palsy. In three patients, there were signs and symptoms of hypocalcemia. In three patients $(10 \%)$, the restoring of biochemical parameters and the resolution of symptoms occurred between 2 weeks and 6 weeks.

Conclusion: Microscopic-assisted thyroidectomy is a feasible and efficacious surgical procedure. It significantly reduces the complications without increasing the operating time in thyroid surgery procedures.

Keywords: Microscopic, Recurrent laryngeal nerve palsy and hypocalcemia, Thyroid

How to cite this article: Gautam HK, Maurya D, Sharma S. Outcome of Microscopic-assisted Thyroid Surgery: An Institutional Retrospective Study. Int J Head Neck Surg 2017;8(4):140-144

\section{Source of support: Nil}

Conflict of interest: None

\footnotetext{
${ }^{1}$ Associate Professor, ${ }^{2-3}$ Junior Resident

${ }^{1-3}$ Department of ENT, GSVM Medical College Kanpur, Kanpur, Uttar Pradesh, India
}

Corresponding Author: Harendra Kumar Gautam, Associate Professor, Department of ENT, GSVM Medical College Kanpur, Kanpur, Uttar Pradesh, India, e-mail: harendragautam34@ yahoo.in

\section{INTRODUCTION}

Thyroid surgery has been characterized in the last years by significant innovations which are well codified and standardized. Although the mortality rate is remarkably reduced $(0.065 \%)$ compared to the early $1900,{ }^{1}$ thyroid surgery is still not free from risk of complications such as those related to the injury of laryngeal nerves and parathyroid glands. ${ }^{2,3}$ The two most common early complications of thyroid surgery are hypocalcemia (20-30\%) and recurrent laryngeal nerve injury $(5-11 \%){ }^{2}$ Bilateral recurrent nerve paralysis resulting in adduction of the vocal cords is a rare life-threatening complication and require emergency management. ${ }^{2}$ To prevent or reduce the incidence of these events, it is of primary importance to have an excellent anatomical knowledge of the neck as well as the application of a meticulous surgical technique. ${ }^{4,5}$ Thus, the rate of these complications is directly related to the extent of thyroidectomy and to a radical thyroid excision (without macroscopic thyroid residual) as well as to the surgeon's experience. ${ }^{6}$ Microscopic-assisted thyroid surgery approach helps surgical performance and prevents complications, especially in interventions, thyroiditis, cancer and cervical lymphadenectomy. ${ }^{4}$ The aim of the present retrospective study was to review the thyroid surgery performed with use of microscopic in a single institution and analyze the outcomes with respect to those reported in the literature with other surgical techniques.

\section{MATERIALS AND METHODS}

This was a retrospective study of 30 patients of clinically diagnosed thyroid nodules. The study was carried out at the Department of ENT of a tertiary-care Medical College and Hospital during the period of years from March 2017 to February 2019. All patients with thyroid nodules underwent provisional diagnoses on the basis of history, physical examination of neck, ultrasound for thyroid gland, serum TSH level, FNAC, and biopsy whenever it was required. Clinical information was collected on sex, age, thyroid disease, and histology. In particular, the incidence of complications related to injuries of inferior laryngeal nerves and parathyroid glands after microscopic assisted thyroid surgery was analyzed. All patients had pre and postoperative mirror vocal cords assessment. 
Outcome of Microscopic-assisted Thyroid Surgery: An Institutional Retrospective Study

To improve the quality of the assessment, all patients had pre and postoperative (about 4-8 weeks) fiber-optic nasolaryngoscopy and voice evaluation. All patients received, from the day after surgery until normalization of serum calcium, $1 \mathrm{~g}$ of calcium carbonate and $1 \mu \mathrm{g}$ of vitamin D supplement for each day; serum calcium and ionized calcium have been dosed at day 1, 2 and 7 after surgery. We consider permanent hypocalcemia a low serum dose of calcium after 6 months to surgery.

\section{RESULTS}

This study included 30 cases of solitary thyroid nodules. Various factors regarding clinical presentation, findings of various investigations, histopathological characteristics and operative treatment had been analyzed. Most of the cases were in the age group of 21-40 years (60\%). The minimum age was 11 years, and maximum age was 60 years. Female preponderance was more than males with the ratio of 5.6:1. (Table 1). All patients (100\%) had swelling over the anterior aspect of the neck, therefore presented with swelling as the chief complaint. Other complaints were dysphagia dyspnea, pain over swelling and hyperthyroidism. Routine thyroid function test (TFT) was done in all patients, and all were found to be in the euthyroid state. Ultrasonography (USG) findings showed 16 cases of thyroid nodule, nine cases showed colloidal cyst and three cases showed multinodular goiter followed by neoplasm in one case. One patient had thyroiditis on USG (Table 2). The most common surgical procedure was hemithyroidectomy which accounts for 28 (93.34\%) (Table 3). There were no cases of operative or postoperative deaths in our series. Due to the clinical relevance of such microscopic-assisted thyroid surgery approach, compared to more traditional ones (Figs 1 and 2), we paid particular attention to the incidence of complications related to injuries of inferior laryngeal nerves and

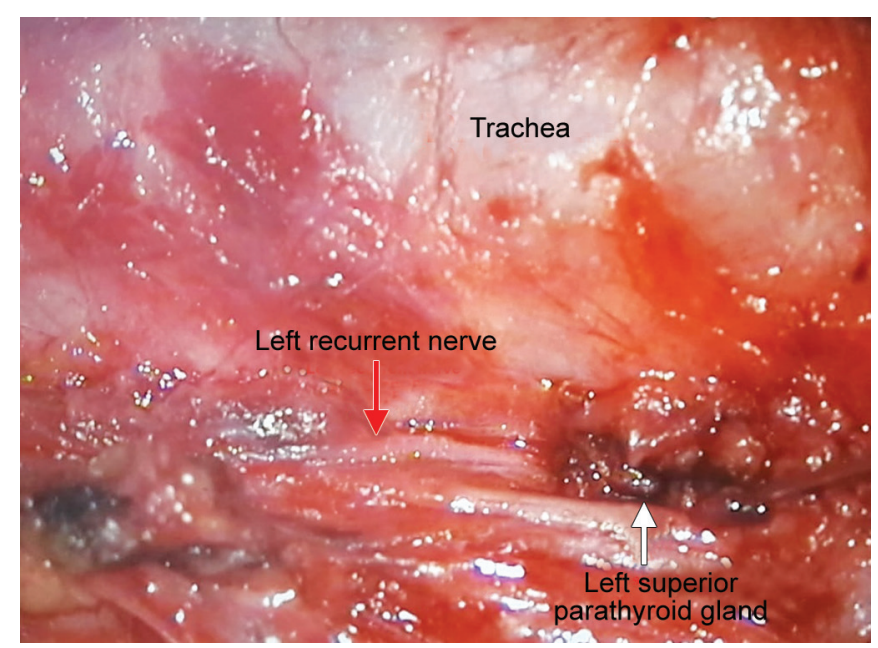

Fig. 1: Intraoperative picture of hemithyroidectomy with microscope
Table 1: Distribution of cases according to age and gender

\begin{tabular}{llll}
\hline Age group (years) & Male & Female & Total \\
\hline $11-20$ & 1 & 8 & 9 \\
$21-30$ & 2 & 5 & 7 \\
$31-40$ & 1 & 10 & 11 \\
$41-50$ & 0 & 2 & 2 \\
$51-60$ & 0 & 1 & 1 \\
$>60$ & 0 & 0 & 0 \\
Total & 4 & 26 & 30 \\
\hline
\end{tabular}

Table 2: Distribution of various conditions by high-resolution ultrasonography findings among cases

\begin{tabular}{ll}
\hline USG diagnosis & Number of cases $(\%)$ \\
\hline Benign thyroid nodule & $16(53.34)$ \\
Colloidal cyst & $9(30)$ \\
Multinodular goiter & $3(10)$ \\
Hashimoto thyroiditis & $1(3.34)$ \\
Thyroid malignancy & $1(3.34)$ \\
Total & $30(100)$ \\
The association among group of USG findings is good at \\
$\mathrm{X}^{2}=>0.60 ; p<0.05$ significant; USG, ultrasonography \\
\hline
\end{tabular}

parathyroid glands. In our series, there were no cases of unilateral or bilateral permanent vocal cord palsy postoperatively, two symptomatic patients with dysphonia were recorded and they had early phoniatric evaluation within 10 days. One of these patient (one of total thyroid operated) had unilateral vocal fold immobility with dysphonia but without dysphagia. Patients with unilateral vocal fold immobility were treated with medical therapy associated with phoniatric speech therapy alongside the rehabilitation speech therapy for better and more rapid recovery of the vocal function. All patients showed complete laryngeal recovery of motility in 6-8 weeks. Regarding the injuries of parathyroid glands, 27 patients (90\%) did not show signs and symptoms of hypocalcemia while three patients (10\%) presented signs of paresthesia and numbness of the fingertips and perioral area and Chvostek's sign positivity, associated with alteration of

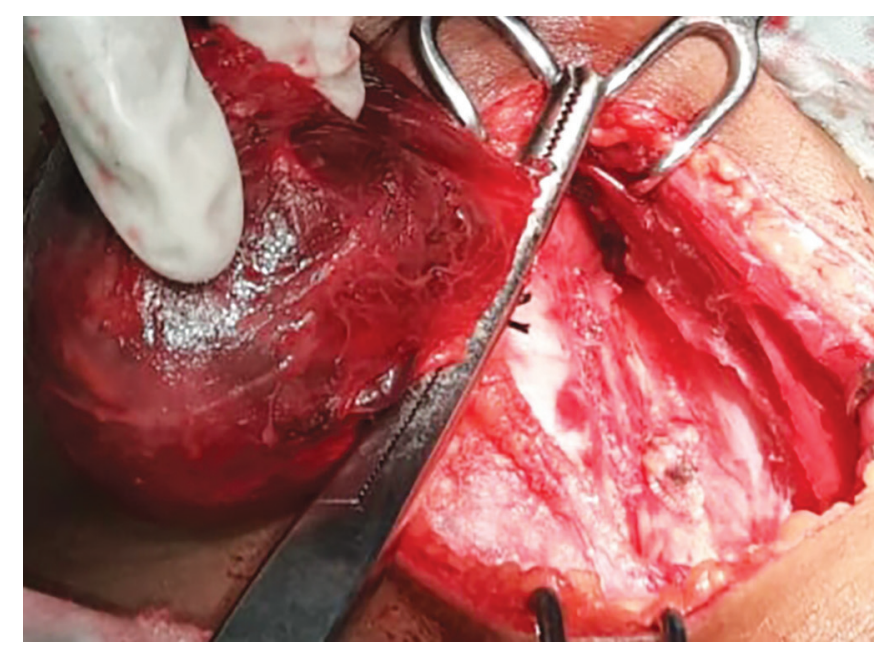

Fig. 2: Intraoperative picture of hemithyroidectomy without microscope 
Table 3: Distribution of various conditions by high-resolution ultrasonography findings and surgical procedure among cases

\begin{tabular}{|c|c|c|c|c|}
\hline \multirow{3}{*}{ USG findings } & \multirow{3}{*}{ Number of cases } & \multicolumn{2}{|c|}{ Surgical procedure, $n(\%)$} & \multirow{3}{*}{ Total thyroidectomy, $n$ (\%) } \\
\hline & & \multicolumn{2}{|c|}{ Hemithyroidectomy } & \\
\hline & & Right & Left & \\
\hline Benign thyroid nodule & 16 & 10 & 6 & 0 \\
\hline Colloidal cyst & 9 & 5 & 4 & 0 \\
\hline Multinodular goiter & 3 & 1 & 1 & 1 \\
\hline Hashimoto thyroiditis & 1 & 1 & 0 & 0 \\
\hline Thyroid malignancy & 1 & 0 & 0 & 1 \\
\hline Total & 30 & $17(56.66)$ & $11(36.66)$ & $2(6.66)$ \\
\hline
\end{tabular}

Table 4: Distribution of various conditions by high-resolution ultrasonography findings and postoperative complication among cases.

\begin{tabular}{|c|c|c|c|c|c|c|c|c|}
\hline \multirow[b]{3}{*}{ USG findings } & \multirow{3}{*}{$\begin{array}{l}\text { Number of } \\
\text { cases }\end{array}$} & \multicolumn{2}{|c|}{$\begin{array}{l}\text { Postoperative } \\
\text { hypocalcemia }\end{array}$} & & stoperative & vocal cord & alsy & \multirow{3}{*}{$\begin{array}{l}\text { Bleeding, esophageal } \\
\text { tracheal, carotid artery } \\
\text { injury and death }\end{array}$} \\
\hline & & \multirow[b]{2}{*}{ Transient } & \multirow[b]{2}{*}{ Definite } & \multicolumn{2}{|c|}{ Unilateral } & \multicolumn{2}{|c|}{ Bilateral } & \\
\hline & & & & Transient & Definite & Transient & Definite & \\
\hline Benign thyroid nodule & 16 & 2 & & & & & & \\
\hline Colloidal cyst & 9 & & & & & & & \\
\hline Multinodular goiter & 3 & 1 & & 1 & & & & \\
\hline Hashimoto thyroiditis & 1 & & & & & & & \\
\hline Thyroid malignancy & 1 & & & 1 & & & & \\
\hline Total & 30 & 3 & & 2 & & & & \\
\hline
\end{tabular}

biochemical parameters of serum calcium and ionized calcium. None of the patients presented Trousseau's sign or tetany. In three patients ( $10 \%$ of all patients), the restoring of the biochemical parameters and the resolution of symptoms had occurred between 2 weeks and 6 weeks (mean 20 days). No morbidity regarding bleeding, bilateral inferior laryngeal nerves palsy, esophagus, trachea, or carotid artery was ever observed in this series (Table 4).

\section{DISCUSSION}

Thyroid enlargement, whether diffuse or nodular leads to a battery of investigations mainly to rule out the possibility of a neoplastic or no neoplastic lesions. Timely intervention in nodular lesions of thyroid can significantly reduce morbidity and mortality. ${ }^{7}$ In the present study, the highest incidence of solitary thyroid nodule was found in the age group of 21-40 years. This result is comparable to the results obtained by Venkatachalapathy et al. ${ }^{8}$ in which the observed female to male ratio was 5.6:1. This female preponderance is reflected in all studies including the present. A similar observation was made by Vyas et al. on 100 patients with thyroid nodule where the ratio was $7: 1 .^{9}$ In this study, all patients (100\%) had swelling over the anterior aspect of the neck. A similar observation was done by Huque et al. on 118 patients with solitary thyroid nodules and found that thyroid swelling was the most common presentation in all cases $(100 \%) .{ }^{10}$ After swelling, other complaints were dyspnea ( $4 \%$ ), dysphagia $(18 \%)$, and pain over the swelling (4\%). Dyspnea and dysphagia are the pressure symptoms, usually present in malignancy but they may be found in the very large nodule. Pain associated with nodule indicates hemorrhage into an adenoma. Routine thyroid profiles were done in each patient to find out the functional status of the thyroid. In this study, all cases were in the euthyroid state. Ultrasound suggested solitary thyroid nodule in 16 patients, colloid nodule/cyst in 9 patients, MNG in 3 patients, and malignancy in 1 patient. One patient had thyroiditis on USG. Walker et al. have shown that the prevalence of multinodularity in clinically solitary thyroid nodules is between $20 \%$ and $40 \%{ }^{11}$ Of all, $96.34 \%$ of patients had benign lesions. Among them, $91.66 \%$ patients had colloid nodule and $5 \%$ had lymphocytic thyroiditis. Out of the 30 patients, one had malignant lesions in the form of papillary carcinoma (1.66\%).

In this study, the most common procedure was hemithyroidectomy (93.34\%) which is almost consistent with the observation of Satihal et al., where hemithyroidectomy (84\%) was the commonest procedure followed by total thyroidectomy $(16 \%){ }^{12}$ We adopted microscopicassisted thyroid surgery, instead of standard surgical technique. As reported above, the two most common early complications of thyroid surgery are hypocalcemia and recurrent laryngeal nerve (RLN) injury, respectively $20-30 \%$ and $5-11 \% .{ }^{2}$ In agreement with the concept that Microscopic-assisted thyroid surgery allows to remarkably reduce the complications of thyroid surgery; in our series, there were no cases of unilateral or bilateral permanent vocal cord palsy, two patients $(0.6 \%)$ had transient unilateral vocal fold immobility with dysphonia without dysphagia, and three of patients reported transient hypocalcemia. The microscopic-assisted thyroid surgery are essential in identifying the thin branches of the inferior laryngeal nerve, which is particularly 
exposed at risk in the last extra laryngeal 2 centimeters (cm), especially to perform a real total excision of thyroid parenchyma. Thus, closed to the Zuckerkandl tubercle and fascial layers, there is a frequent site of injury due to transection, clamping, ligation, traction, diathermy injury and ischemia. ${ }^{13}$ At that level, great caution must be taken in ligating and dividing such structures, in particular the arterial branches located behind the nerve, to prevent the bleeding whose hemostasis would damage the nerve. For this purpose, we used microscopic-assisted thyroid surgery ligatures with absorbable suture $4-0$ and $6-0$ so as to avoid thermal nerve damage and local edema, avoiding the use of bipolar diathermy. To ensure the integrity of the external branch of the superior laryngeal nerve, we paid special attention during dissection of upper vascular pole, tying first the anterior branch of the artery and then the posterior branches, leaving no residual thyroid tissue at ligatures, in agreement with the literature. ${ }^{14}$ Particular care should be taken during the dissection of the recurrent nerve near the trunk of the inferior thyroid artery, ${ }^{15}$ avoiding devascularization of the parathyroid glands and dissecting the arterial branches with thin ties near their entry into the gland. A technique that has been proposed to prevent RLN injuries and to aid nerve localization before visualization is the intraoperative neuromonitoring (IONM). ${ }^{16}$ According to some authors, IONM reduces the rate of transient inferior laryngeal nerve palsy, it is useful in the case of thyroid re-surgery, and can help in the identification of the RLN during dissection before visualization. ${ }^{17}$ However, the role and the utility of IONM during thyroid surgery is still under debate ${ }^{18}$ and, at the moment, there is no consensus regarding the prevention of RLN injury. ${ }^{19}$ In our experience, the use of IONM did not reduce the risks of laryngeal nerves injury (data not shown), suggesting that IONM does not improve the surgeon's capability to identify and dissect the nerve. In comparison, the advantages of the microscopic vision are clearly evident in video-assisted surgery, and recently it has been proposed to use three-dimensional minimally invasive video-assisted thyroidectomy for better perception of depth and easy recogntion of anatomic structures, especially concerning the upper and lower vascular pedicle, the parathyroids and the laryngeal nerves. ${ }^{20}$ Microscope-assisted thyroidectomy has also been championed for its ergonomic benefits to the surgeon. The operating microscope has been proposed as a method to maintain an upright posture with neutral cervical position during thyroid surgery, so reducing occupational musculoskeletal risk. ${ }^{21}$ The authors, though, noted a significant increase in operating time especially in case of lateral neck dissection. Nielsen et al. reported slight increases in operating time ${ }^{22}$ while, in a more recent prospective controlled trial, a direct comparison of thyroidectomy performed with and without the operating microscope found no significant differences in operating time. ${ }^{23}$ Moreover, the use of the operating microscope also allows recording which has obvious benefits for both teaching purposes and for a medico-legal standpoint. ${ }^{4}$

\section{CONCLUSION}

Microscopic-assisted thyroidectomy is a feasible and efficacious surgical procedure. It significantly reduces the complications without increasing the operating time in thyroid surgery procedures.

\section{REFERENCE}

1. Gómez-Ramírez J, Sitges-Serra A, Moreno-Llorente P, et al. Mortality after thyroid surgery, insignificant or still an issue Lange. Becks Arch Surg. 2015; 400:517-522.

2. Christou N, Mathonnet M. Complications after total thyroidectomy. J Visc Surg. 2013; 150:249-256.

3. Selberherr A, Scheuba C, Riss P, et al. Postoperative hypoparathyroidism after thyroidectomy: Efficient and cost-effective diagnosis and treatment. Surgery. 2015; 157:349-353.

4. Williams SP, Wilkie MD, Tahery J. Microscope-assisted thyroidectomy: Our experience in one hundred and twenty-one consecutive cases. Clin Otolaryngol. 2014; 39:289-315.

5. Trésallet $\mathrm{C}$, Chigot JP, Menegaux F. How to prevent recurrent nerve palsy during thyroid surgery? Annal Chir. 2006; 131:149-153.

6. Hayward NJ, Grodski S, Yeung M, et al. Recurrent laryngeal nerve injury in thyroid surgery: a review. ANZ J Surg. 2013; 83:15-21.

7. Chandanwale S. Clinicopatholological correlation of thyroid nodules. Int J Pharm Biomed Sci. 2012; 3(3):97-102.

8. Venkatachalapathy TS, Sreeramulu PN. A prospective study of clinical, sonological and pathological evaluation of thyroid nodule. Thyroid Disorders Ther. 2012; 1:2.

9. Vyas CS, Vijayvargiya SC. A study of thyroid swelling with clinicopathological parameters. Int J Biol Med Res. 2013; 4(2):3250-3252.

10. Huque SN, Ali MI, Huq MM, Rumi SN, Sattar MA, Khan AM. Histopathological pattern of malignancy in solitary thyroid nodule. Bangladesh J Otorhinolaryngol. 2012; 18(1):5-10.

11. Walker J, Findlay D, Amar SS, Small PG, Wastie ML, Pegg CA, et al. A prospective study of thyroid ultrasound scan in the clinically solitary thyroid nodule. Br J Radiol 1985; 58:6179.

12. Satihal SN, Palled ER. A study of various clinical presentation of solitary thyroid nodule at tertiary care center. Med Pulse Int Med J. 2014; 1(1):30-32.

13. Serpell JW. New operative surgical concept of two fascial layers enveloping the recurrent laryngeal nerve. Ann Surge Oncol.2010; 17:1628-1636.

14. Monfared A, Gorti G, Kim D. Microsurgical anatomy of the laryngeal nerves as related to thyroid surgery. Laryngoscope. 2002; 112:386-392.

15. Yalçin B. Anatomic configurations of the recurrent laryngeal nerve and inferior thyroid artery. Surgery. 2006;139:181-187.

16. Duclos A, Lifante JC, Ducarroz S, et al. Influence of Intraoperative Neuromonitoring on Surgeons' Technique During Thyroidectomy. World J Surg. 2011;35:773-778. 
17. Dralle H, Sekulla C, Haerting J, et al. Risk factors of paralysis and functional outcome after recurrent laryngeal nerve monitoring in thyroid surgery. Surgery. 2004; 136:1310-1322.

18. Pisanu A, Porceddu G, Podda M, et al. Systematic review with meta-analysis of studies comparing intraoperative neu-romonitoring of recurrent laryngeal nerves versus visualiza-tion alone during thyroidectomy. J Surg Res. 2014; 188(1):152-161

19. Dionigi G, Van SlyckeS, Boni L, et al. Limits of Neuromonitoring in Thyroid Surgery. Ann Surg. 2013; 258:E1-E2.

20. Mercante G, Battaglia P, Manciocco V, et al. Three-dimensional minimally invasive video-assisted thyroidectomy: preliminary report. JECCR. 2013; 32:78.
21. Davidson BJ, Guardiani E, Wang A. Adopting the operating microscope in thyroid surgery: safety, efficiency, and ergonomics. Head Neck. 2010; 32:154-159.

22. Nielsen TR, Andreassen UK, Brown CL, et al. Microsurgical technique in thyroid surgery. A 10-year experience. Laryngol Otol.1998; 112:556-560.

23. Seven H, Calis AB, Vural C, Turgut S. Microscopic thyroidectomy: a prospective controlled trial. Eur Arch Otorhinolaryngol.2005; 262:41-44. 